\title{
NUEVAS CITAS PARA TRES HÍBRIDOS DE ASPLENIUM (ASPLENIACEAE, PTERIDOPHYTA) EN LA PENÍNSULA IBÉRICA
}

\author{
Alberto HERRERO NIETO y Carmen PRADA MORAL
}

\begin{abstract}
RESUMEN. Nuevas citas para tres híbridos de Asplenium (Aspleniaceae, Pteridophyta) en la Península Ibérica. Se dan a conocer nuevas localidades en la Península Ibérica para los siguientes híbridos de Asplenium: Asplenium x protomajoricum nothosubsp. protomajoricum (=A. fontanum subsp. fontanum $\mathrm{x}$ A. petrarchae subsp. bivalens), A. x recoderi nothosubsp. recoderi (= A. fontanum subsp. fontanum $\times$ A. ruta-muraria subsp. ruta-muraria) y A. x sleepiae nothosubsp. sleepiae (= A. obovatum subsp. lanceolatum $\mathrm{x}$ A. foreziense). Se aportan los datos del análisis citológico y se describen los principales caracteres morfológicos de las frondes.
\end{abstract}

Palabras clave. Pteridophyta, Aspleniaceae, Asplenium, híbridos, citología, morfología, corología, Península Ibérica.

ABSTRACT. New records for three Asplenium hybrids (Aspleniaceae, Pteridophyta) in the Iberian Peninsula. New Iberian localities are reported for the following Asplenium hybrids: Asplenium $x$ protomajoricum nothosubsp. protomajoricum (= A. fontanum subsp. fontanum x A. petrarchae subsp. bivalens), A. x recoderi nothosubsp. recoderi ( $=$ A. fontanum subsp. fontanum x A. ruta-muraria subsp. ruta-muraria) and A. $x$ sleepiae nothosubsp. sleepiae (= A. obovatum subsp. lanceolatum $\mathrm{x}$ A. foreziense). Results of the cytological analysis and a description of the main morphological features of the fronds are given.

Key words. Pteridophyta, Aspleniaceae, Asplenium, hybrids, cytology, morphology, geographical distribution, Iberian Peninsula.

\section{INTRODUCCIÓN}

Dentro de los pteridófitos, el género Asplenium es uno de los que con más facilidad produce híbridos. En Europa, donde este género ha sido extensamente estudiado, se reconocen más de 50 híbridos (Reichstein, 1981), que aparecen con cierta frecuencia en aquellas zonas donde los parentales están en proximidad.

Estos híbridos se detectan en el campo por presentar normalmente una morfología intermedia con respecto a sus parentales y un 
contenido esporangial abortivo. La confirmación de su origen es posible, en la mayor parte de los casos, mediante el análisis del comportamiento meyótico de los cromosomas en las células madres de las esporas.

Para la revisión taxonómica que estamos llevando a cabo en el grupo de los Asplenium ibéricos de fronde lanceolada (A. fontanum, $A$. foreziense y $A$. obovatum), se ha realizado una amplia prospección del territorio peninsular, habiendo encontrado en varios puntos híbridos relacionados con este grupo, de los que hemos realizado el estudio que se expone a continuación.

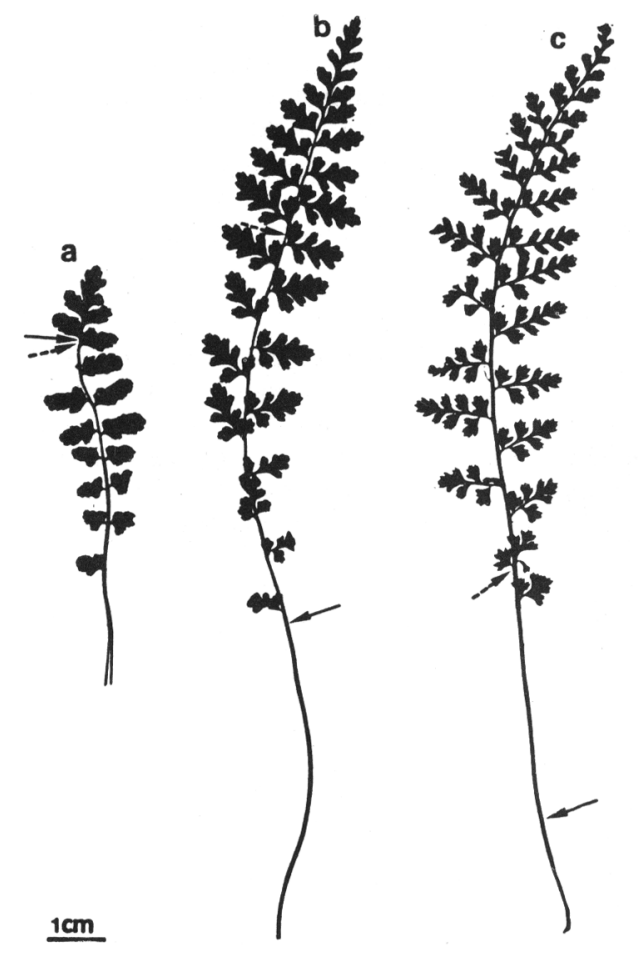

Figura 1. Siluetas de las frondes: a, Asplenium petrarchae subsp. bivalens; b, A. x protomajoricum nothosubsp. protomajoricum; c, A. fontanum subsp. fontanum. $(\rightarrow)$ : Nivel que alcanza el color marrón oscuro en el pecíolo y raquis por la cara adaxial, y $(-\rightarrow)$ por la cara abaxial de la fronde.

\section{MATERIAL Y MÉTODOS}

El estudio citológico se ha realizado en esporangios fijados directamente en el campo en una mezcla de alcohol absoluto-ácido acético (3:1); en algunos casos la fijación se llevó a cabo en el laboratorio, tras permanecer las frondes varias horas en la nevera. Para la visualización de los cromosomas y su posterior estudio, los esporangios fueron teñidos con hematoxilina de Wittman y montados en medio Hoyer diluido para obtener preparaciones semipermanentes.

Los detalles de la morfología de las pinnas y de los indusios se obtuvieron a partir de material rehidratado y aclarado con hipoclorito sódico al $5 \%$.

La procedencia del material estudiado se indica en cada caso.

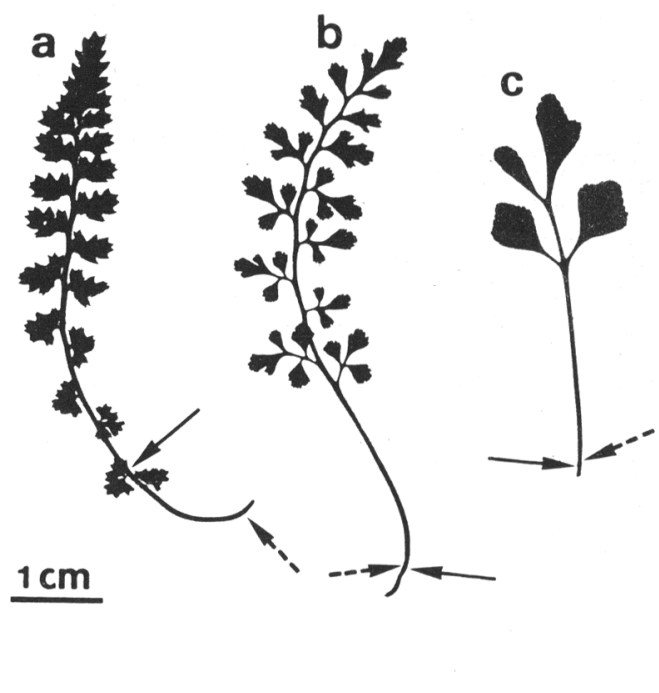

Figura 2. Siluetas de las frondes: a, Asplenium fontanum subsp. fontanum; b, A. x recoderi nothosubsp. recoderi; c, A. ruta-muraria subsp. ruta-muraria. $(\rightarrow)$ Nivel que alcanza el color marrón oscuro en el pecíolo y raquis por la cara adaxial, y $(--\rightarrow)$ por la cara abaxial de la fronde. 


\section{RESULTADOS Y DISCUSIÓN}

Asplenium $x$ protomajoricum Pangua \& Prada nothosubsp. protomajoricum (= Asplenium fontanum (L.) Bernh. subsp. fontanum $\mathrm{x} A$. petrarchae (Guérin) DC. subsp. bivalens (D.E. Mey.) Lovis \& Reichst.).

ALICANTE: Campell, vall de Laguart, 30SYH59, c. $400 \mathrm{~m}$, grieta de roca caliza, 18VI-1995, A. Herrero, CAM5.

El ejemplar encontrado se localizaba conviviendo con sus parentales. Además de los escasos pelos pluricelulares comunes en muchas especies de Asplenium, presenta numerosos pelos unicelulares en la lámina, pecíolo y raquis, característica que es propia de A. petrarchae subsp. bivalens.
Las frondes tienen una morfología similar a la de $A$. fontanum (fig. 1 a-c), si bien las pinnas son menos incisas y los dientes no tan cuspidados (fig. 4 a-c); el pecíolo y la mitad inferior del raquis son de color marrón oscuro, siendo en este carácter intermedio el híbrido con respecto a sus parentales (fig. 1 a-c). El raquis sin embargo es de sección circular, como ocurre en $A$. petrarchae subsp. bivalens y no aplanado y acanalado, como es el caso en $A$. fontanum. No se aprecian diferencias en los indusios de los tres táxones (fig. $5 \mathrm{a}-\mathrm{c}$ ).

Se ha podido realizar el análisis de la meyosis en cuatro células madres de las esporas, que presentaron $n=72^{1}$ en tres de ellas (fig. 6 a y a') y $n=70^{\mathrm{I}} 1^{\mathrm{II}}$ en la otra.

Este híbrido diploide ha sido citado únicamente del Levante español; hasta ahora se habían recolectado dos (Pangua et al., 1992)
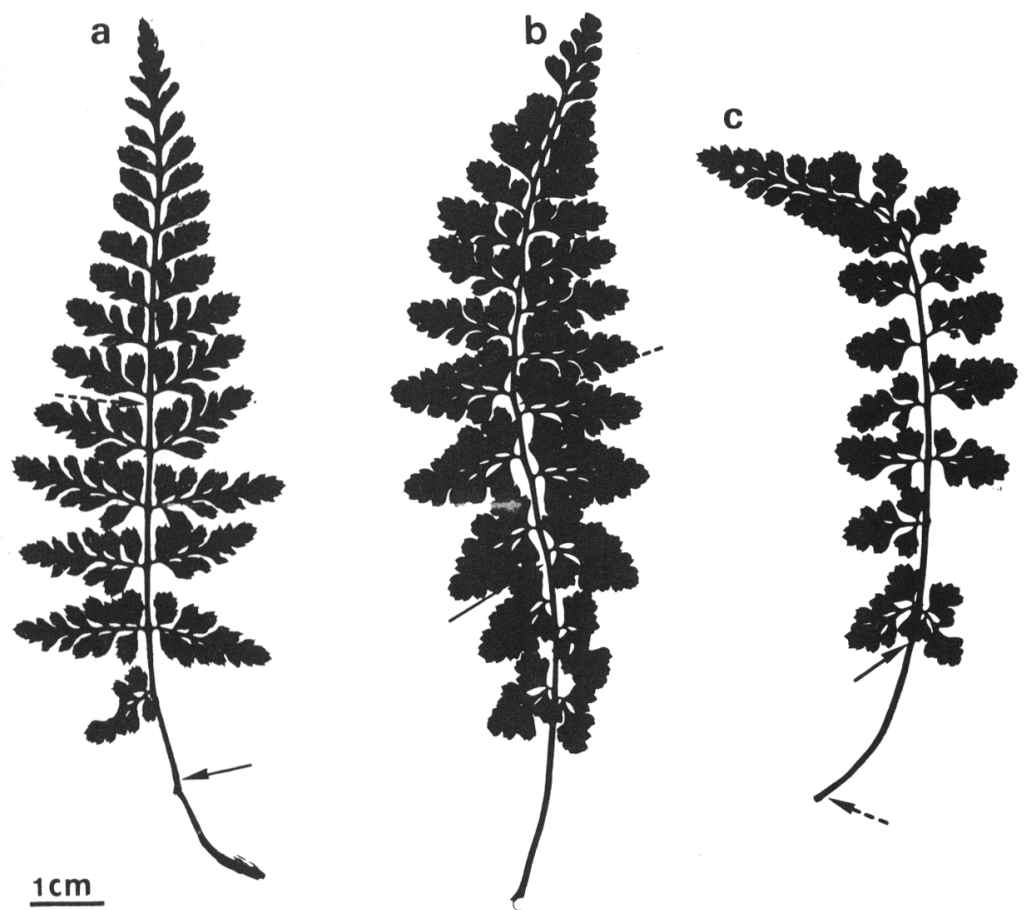

Figura 3. Siluetas de las frondes: a, Asplenium obovatum subsp. lanceolatum; b, A. $x$ sleepiae nothosubsp. sleepiae; c, A. foreziense. $(\rightarrow)$ Nivel que alcanza el color marrón oscuro en el pecíolo y raquis por la cara adaxial, y $(--\rightarrow)$ por la cara abaxial de la fronde. 
y cinco individuos (Pérez Carro y Fernández Areces, 1992), en la provincia de Valencia. Por las descripciones del híbrido que estos autores hacen, y a la vista del nuevo individuo encontrado por nosotros, podemos afirmar que la pubescencia de las frondes es extremadamente variable, habiéndose encontrado plantas con la lámina y el pecíolo prácticamente glabros, otras con la superficie abaxial de las pinnas provista de pelos glandulares dispersos y otras con abundantes pelos en toda la fronde.

En el ejemplar aquí estudiado se han observado algunas diplósporas. Reichstein (1981) señala que existe gran variación en la capacidad de las especies diploides para formar híbridos y en el potencial de tales híbridos para duplicar posteriormente sus cromosomas y originar alopoliploides. En este caso, la duplicación de los cromosomas en el híbrido parece ser relativamente fácil dado el contenido en diplósporas denunciado en los ejemplares hasta ahora localizados; este hecho probablemente está relacionado con la presencia en esa región del alopoliploide derivado, A. majoricum Litard., recientemente recolectado en Valencia (Pérez Carro y Fernández Areces, 1992) y cuya presencia en la provincia de Alicante fue indicada por Pangua et al. (1992) basándose en material de herbario recolectado por Rigual y señalado por este autor bajo A. lanceolatum Huds. var. valentinum (Pau) Knoch (Rigual, 1972).

Asplenium $x$ recoderi Aizpuru \& Catalán nothosubsp.recoderi (= Asplenium fontanum a
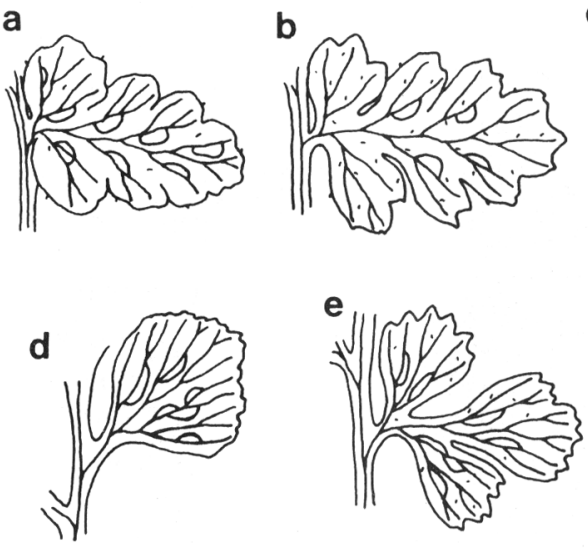
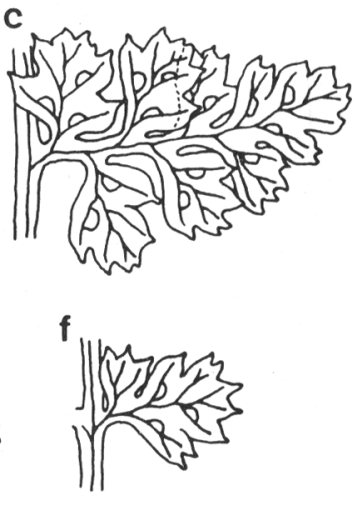
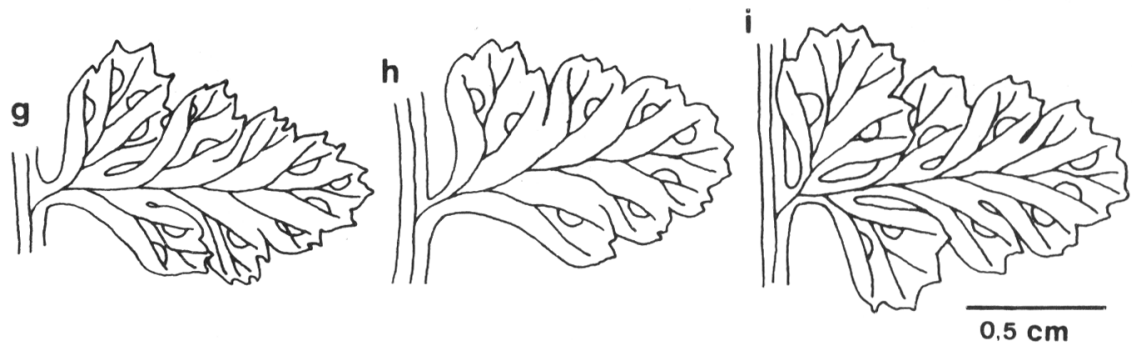

Figura 4. Pinna media de las frondes: a, Asplenium petrarchae subsp. bivalens; b, A. x protomajoricum nothosubsp. protomajoricum; c, A. fontanum subsp. fontanum; d, A. ruta-muraria subsp. ruta-muraria; e, A. $x$ recoderi nothosubsp. recoderi; f, A. fontanum subsp. fontanum; g, A. obovatum subsp. lanceolatum; h, A. x sleepiae nothosubsp. sleepiae; i, A. foreziense. 
(L.) Bernh. subsp. fontanum x A. rutamuraria L. subsp. ruta-muraria).

HUESCA: Benasque, valle de Estós, $31 \mathrm{TBH} 92$, c. $1300 \mathrm{~m}$, grieta de roca caliza, 24VIII-1996, J.L. Castillo \& A. Herrero, EST2.

El individuo que hemos recolectado presenta las frondes de morfología intermedia entre las de sus parentales (fig. 2 a-c), con la lámina lanceolada como en A. fontanum y las pinnas basales divididas de forma pinnada, con pinnulas flabeladas muy semejantes a las de A. ruta-muraria, pero presentando pequeños dientes agudos bien definidos y abundantes
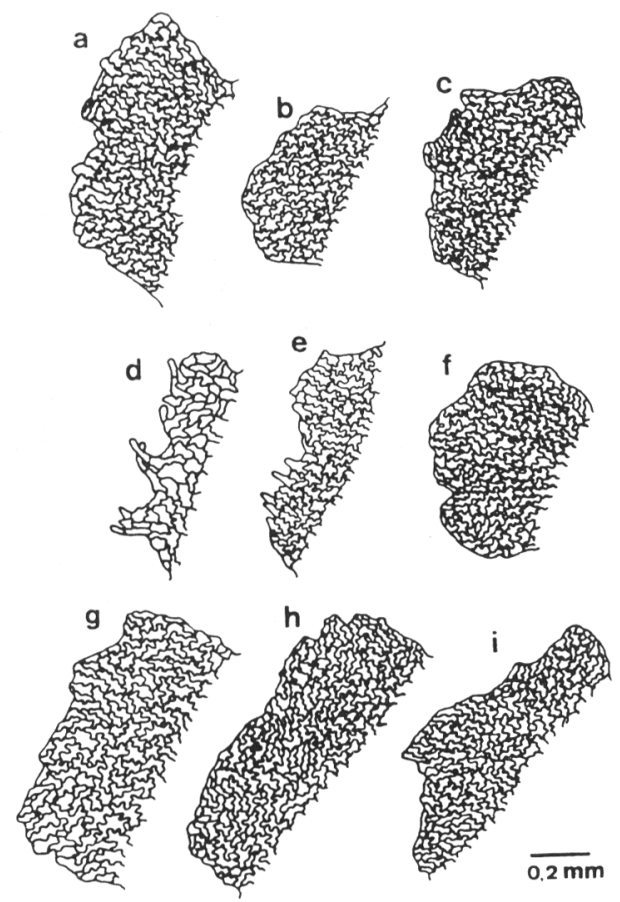

Figura 5. Modelos de las células de los indusios: a, Asplenium petrarchae subsp. bivalens; b, A. $x$ protomajoricum nothosubsp. protomajoricum; c, $A$. fontanum subsp. fontanum; d, A. ruta-muraria subsp. ruta-muraria; e, A. x recoderi nothosubsp. recoderi; f, A. fontanum subsp. fontanum; g, A. obovatum subsp. lanceolatum; h, A. x sleepiae nothosubsp. sleepiae; i, A. foreziense. pelos pluricelulares (fig. 4 d-f). Los caracteres del indusio son también intermedios en este híbrido, lo que se manifiesta principalmente por la presencia de algunas fimbrias en el borde del indusio al igual que ocurre en $A$. ruta-muraria y por el tamaño y tipo de células, semejantes a las de A. fontanum (fig. 5 d-f).

En las dos células madres de las esporas en que ha sido posible observar la meyosis, se ha obtenido $n=$ c. $36^{\mathrm{I}}$ y $36^{\mathrm{II}}$ (fig.6 b y b').

Este híbrido triploide ha sido citado en el Pirineo navarro (Aizpuru y Catalán, 1986) y en la Sierra de Albarracín (Pérez Carro y Fernández Areces, 1996), habiéndose encontrado un solo individuo en cada caso, al igual que en nuestra recolección, en que el único ejemplar hallado se encontraba conviviendo con sus parentales. Si bien hasta ahora este híbrido se ha encontrado ocasionalmente, el hecho de que $A$. fontanum y A. ruta-muraria se desarrollen sobre sustratos básicos y sus áreas de distribución se solapen, hace pensar que posiblemente se encuentre en otros puntos del área en que esos dos táxones coinciden.

Asplenium x sleepiae Badré \& Boudrie nothosubsp. sleepiae (=Asplenium obovatum subsp. lanceolatum (Fiori) P. Silva x A. foreziense Legrand ex Hérib.).

GERONA: El Port de la Selva, Puig dels Bufadors, 31TEG28, c. $400 \mathrm{~m}$, en grieta de roca esquistosa, 9-XII-1994, G. Aragón, A. Herrero, I. Martínez \& A. Pujol, BUF21 y BUF31.

TERUEL: Albarracín, Sierra de Albarracín, barranco del Navazo, 30TXK37, c. $1250 \mathrm{~m}$, grieta de areniscas del Buntsandstein, 18-V-1996, G. Aragón, A. Herrero \& I. Martinez, CAB7.

Este híbrido lo hemos encontrado en dos localidades, una de ellas con dos individuos. Tanto los parentales como el híbrido presentan 


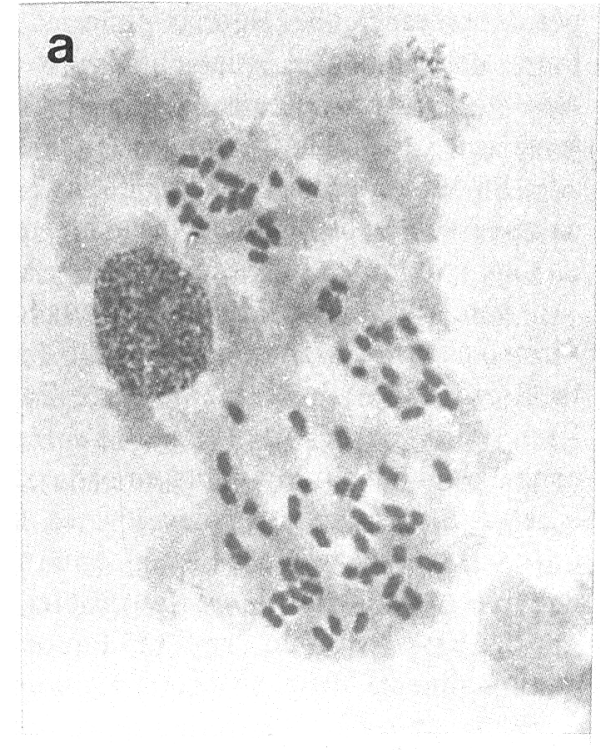

$a^{5}$

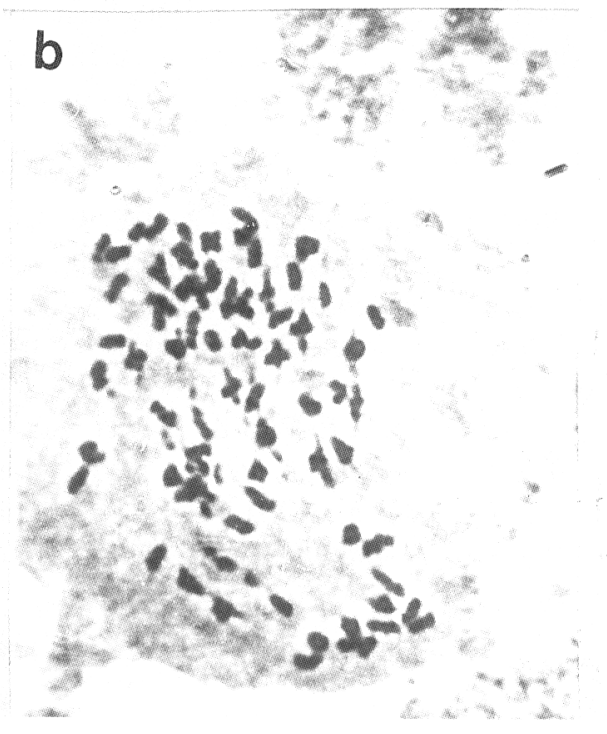

$b^{\prime}$

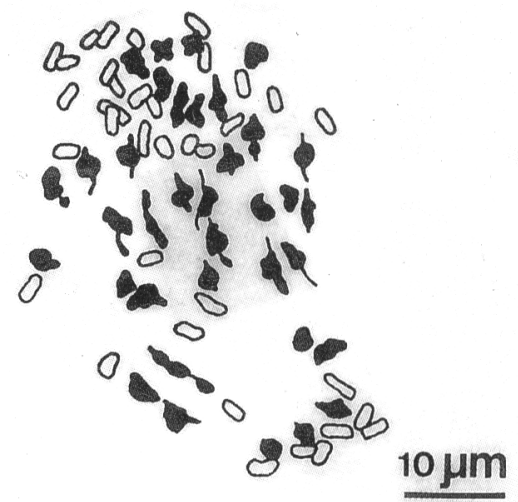

Figura 6. Fotografías y diagramas explicativos de la meyosis de las células madres de las esporas: a y a', Asplenium x protomajoricum nothosubsp. protomajoricum, individuo CAM $5, n=c$. $72^{\text {L' }}$ b y b', $A$. $x$ recoderi nothosubsp. recoderi, individuo EST2, $n=$ c. $36^{1} 36^{11}$. 
a
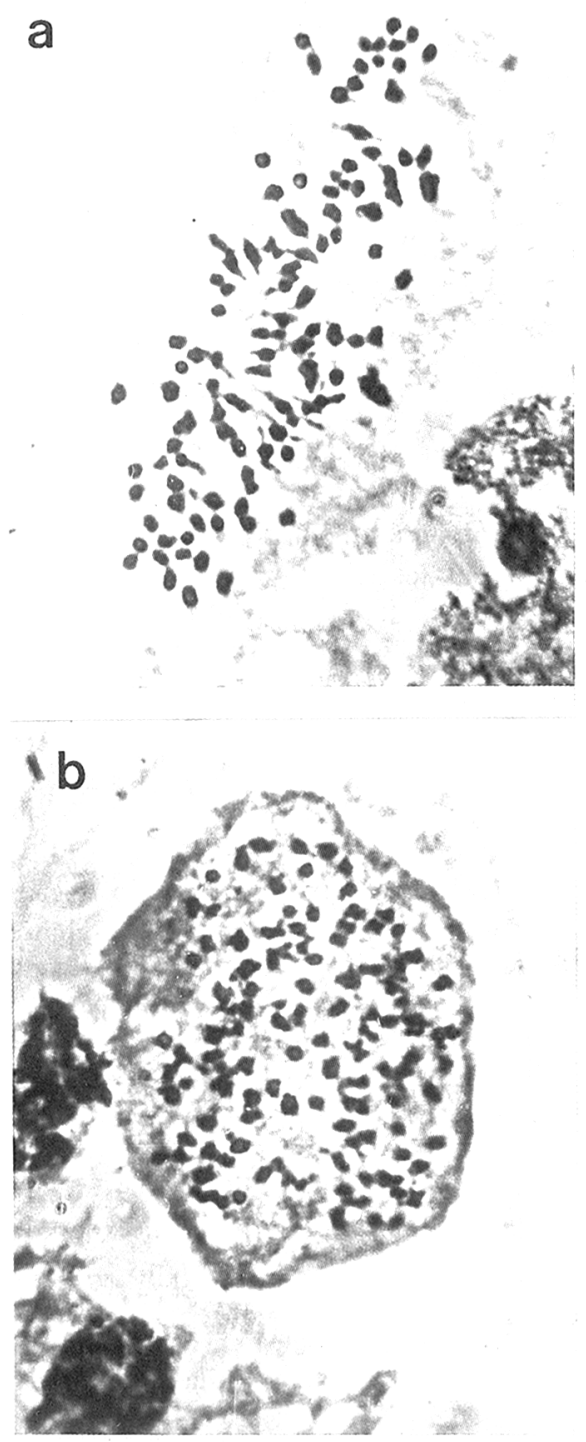

$a^{\prime}$

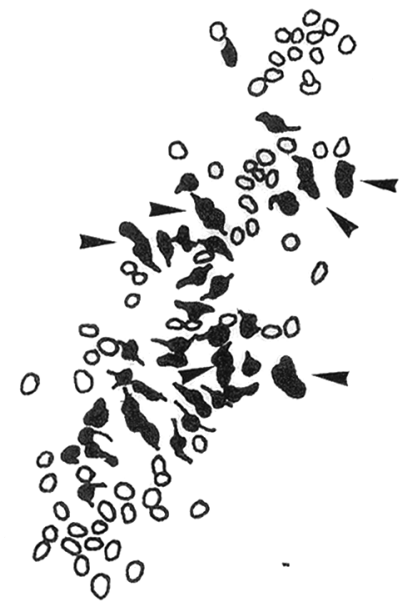

$b^{\prime}$

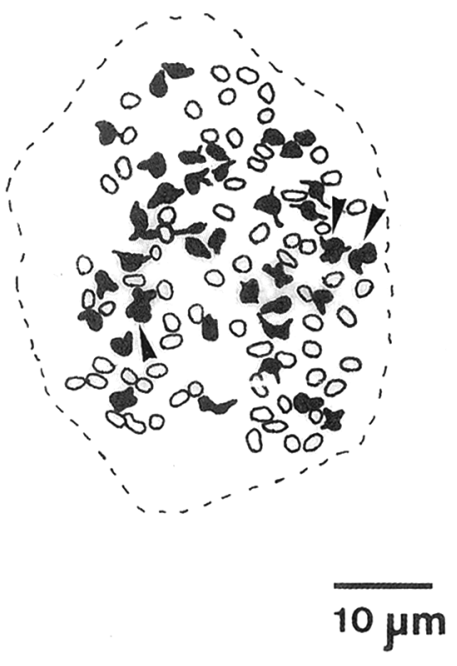

Figura 7. Fotografías y diagramas explicativos de la meyosis de las células madres de las esporas: a y a', Asplenium $x$ sleepiae nothosubsp. sleepiae, individuo BUF21, $n=c$. $66^{1} 30^{11} 6^{\text {III }}$ b y b', Asplenium $x$ sleepiae nothosubsp. sleepiae, individuo $\mathrm{CAB} 7, n=\mathrm{c} .69^{\mathrm{I}} 33^{\mathrm{II}} 3^{\mathrm{III}}$. Las flechas indican los trivalentes. 
una morfología muy parecida en lo que se refiere a la forma y grado de disección de la lámina (fig. 3 a-c), observándose algunas diferencias en las características de los dientes de las pínnulas, que son divergentes y cortamente mucronados en A. foreziense, aproximados y largamente cuspidados en $A$. obovatum subsp. lanceolatum e intermedios en el híbrido (fig. 4 g-i)

El análisis de la meyosis en los individuos estudiados ha dado como resultado $n=$ c. $66^{\mathrm{I}}$ $30^{\mathrm{II}} 6^{\mathrm{III}}$ (fig. 7 a y a') y $n=$ c. $65^{\mathrm{I}} 32^{\mathrm{II}} 5^{\mathrm{III}}$ en el individuo BUF 21 , y $n=$ c. $69^{\mathrm{I}} 33^{\mathrm{II}} 3^{\mathrm{III}}$ en el individuo $\mathrm{CAB} 7$ (fig. 7, b y b').

Debido a que en el híbrido se presentan tres genomas provenientes de $A$. obovatum subsp. obovatum pueden aparecer un número variable de trivalentes, habiéndose encontrado en los individuos hasta ahora estudiados citológicamente, de 0 a 15 trivalentes.

Este híbrido tetraploide fue obtenido de forma artificial por Sleep (1983), y hasta ahora se ha encontrado de forma natural en Francia y en España. En Francia se conoce de numerosas localidades correspondientes a varios departamentos (Badré et al., 1981; Rasbach et al., 1991; Bouzillé y Boudrie, 1991; Boudrie, 1996); en España Sáez et al. (1993) lo habían encontrado en Gerona, en la misma localidad que nosotros. En el Herbario de la Facultad de Ciencias de Málaga hemos estudiado un pliego (MGC 12288) que contiene dos individuos procedentes de Sierra Nevada, del río Lanjarón; uno de los individuos corresponde a $A$. obovatum subsp. lanceolatum y el otro, con el contenido esporangial abortivo y morfología intermedia entre $A$. obovatum subsp. lanceolatum y $A$. foreziense, lo atribuimos a este híbrido.

En todos los casos las plantas se desarrollaban sobre sustrato silíceo (granitos, pizarras, esquistos, areniscas), donde crecen habitualmente sus parentales.

A. foreziense es el alotetraploide derivado de A. fontanum y A. obovatum subsp. obovatum.
A Asplenium obovatum subsp. lanceolatum se le supone el autotetraploide derivado de $A$. obovatum subsp. obovatum o de una forma con cromosomas homólogos a éste (Sleep, 1983). La estrecha relación entre estos táxones hace que presenten una morfología muy similar, diferenciándose normalmente por el grado de división de la lámina y por las características de los dientes de las pinnas (Pangua et al., 1990), pero esta diferenciación no siempre es fácil dada la variabilidad de los dos táxones. La morfología del híbrido es también variable, asemejándose unas veces más a uno de sus parentales y otras veces al otro.

Al ser los parentales muy próximos genéticamente, parece que es frecuente la aparición de este híbrido allí donde conviven sus parentales.

\section{BIBLIOGRAFÍA}

AIZPURU, I. y P. CATALÁN -1986- Asplenium x recoderi, nothosp. nov. Anales Jard. Bot. Madrid 42(2): 531-532.

BADRÉ, F., M. BOUDRIE, R. PRELLI \& J. SCHNELLER - 1981- Asplenium x sleepiae (A. billotii $x$ A. foreziense) et Asplenium $x$ bouharmontii (A. obovatum x A. onopteris), hybr. nov. (Aspleniaceae, Pteridophyta). Bull. Mus. Hist. Nat. Paris, Adansonia 4: 473-481.

BOUDRIE, M. - 1996-Observations pteridologiques dans le departement des Pyrenées-Orientales. Le Monde des Plantes 456: 1-8.

BOUZILLÉ, J.B.\& M. BOUDRIE -1991- Cinquième journée: Samedi 14 avril: les valléés des Albères. Bull. Soc. Bot. Centre-Ouest, n.s. 22: 365-372.

PANGUA, E., C. PRADA, A. CASTILLO y A.E. SALVO -1990- Asplenium obovatum Viv. en la Península Ibérica. In: J. Rita (Ed.), Taxonomía, Biogeografía y Conservación de Pteridófitos 191-208.

PANGUA, E., C. PRADA, S. PAJARÓN \& A.E. SALVO -1992- A new Asplenium hybrid from Valencia (Spain) related to A. majoricum Litard. Bot. J. Linn. Soc. 108: 1-13.

PÉREZ CARRO, F.J. y M.P. FERNÁNDEZ ARECES -1992- Asplenium x protomajoricum hybrid. nov. (A. fontanum subsp. fontanum x A. 
petrarchae subsp. bivalens) y precisiones corológicas sobre A. majoricum en el Levante español. Anales Jard. Bot. Madrid 49(2): 187 194.

PÉREZ CARRO, F.J. y M.P. FERNÁNDEZ ARECES - 1996- Híbridos del género Asplenium L. (Aspleniaceae) en la Península Ibérica. Anales Jard. Bot. Madrid 54(1): 106-125.

RASBACH, H., K. RASBACH, T. REICHSTEIN \& H.W. BENNERT - 1991- Asplenium trichomanes subsp. coriaceifolium, a new subspecies and two new intraspecific hybrids of the $A$. trichomanes complex (Aspleniaceae, Pteridophyta). II. Description and illustrations. With an appendix on pairing behaviour of chromosomes in fern hybrids. Wildenowia 21: 239-261.

REICHSTEIN, T. -1981- Hybrids in European Aspleniaceae (Pteridophyta). Bot. Helvetica 91: 89-139.

RIGUAL, A. -1972- Flora y vegetación de la provincia de Alicante. Alicante.
SÁEZ, L., P. CUBAS y J.A. ROSSELLÓ -1993Asplenium x sleepiae and A. obovatum subsp. obovatum (Aspleniaceae, Pteridophyta) from Cap de Creus, NE Spain. Nova Hedwigia 57(34): 437-443.

SLEEP, A. -1983- On the genus Asplenium in the Iberian Peninsula. Acta Bot. Malacitana 8: 1146.

Aceptado para su publicación en Abril de 1997

Dirección de los autores. Departamento de Biología Vegetal I, Facultad de Biología, Universidad Complutense. 28040, Madrid. 